\title{
HABERTOR: An Efficient and Effective Deep Hatespeech Detector
}

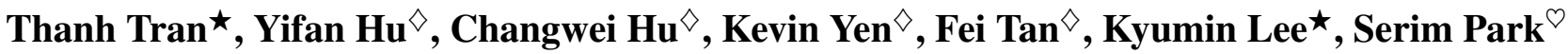 \\ ${ }^{\star}$ Worcester Polytechnic Institute, $\diamond$ Yahoo! Research, ${ }^{\odot}$ Twitter \\ \{tdtran, kmlee\}@wpi.edu \\ \{yifanhu, changweih, kevinyen, fei.tan\}@verizonmedia.com \\ serimpetwitter.com
}

\begin{abstract}
We present our HABERTOR model for detecting hatespeech in large scale user-generated content. Inspired by the recent success of the BERT model, we propose several modifications to BERT to enhance the performance on the downstream hatespeech classification task. HABERTOR inherits BERT's architecture, but is different in four aspects: (i) it generates its own vocabularies and is pre-trained from the scratch using the largest scale hatespeech dataset; (ii) it consists of Quaternionbased factorized components, resulting in a much smaller number of parameters, faster training and inferencing, as well as less memory usage; (iii) it uses our proposed multisource ensemble heads with a pooling layer for separate input sources, to further enhance its effectiveness; and (iv) it uses a regularized adversarial training with our proposed finegrained and adaptive noise magnitude to enhance its robustness. Through experiments on the large-scale real-world hatespeech dataset with $1.4 \mathrm{M}$ annotated comments, we show that HABERTOR works better than 15 state-ofthe-art hatespeech detection methods, including fine-tuning Language Models. In particular, comparing with BERT, our HABERTOR is $4 \sim 5$ times faster in the training/inferencing phase, uses less than $1 / 3$ of the memory, and has better performance, even though we pretrain it by using less than $1 \%$ of the number of words. Our generalizability analysis shows that HABERTOR transfers well to other unseen hatespeech datasets and is a more efficient and effective alternative to BERT for the hatespeech classification.
\end{abstract}

\section{Introduction}

The occurrence of hatespeech has been increasing (Barna, 2019). It has become easier than before to reach a large audience quickly via social media, causing an increase of the temptation for inappropriate behaviors such as hatespeech, and potential damage to social systems. In particular, hatespeech interferes with civil discourse and turns good people away. Furthermore, hatespeech in the virtual world can lead to physical violence against certain groups in the real world ${ }^{12}$, so it should not be ignored on the ground of freedom of speech.

To detect hatespeech, researchers developed human-crafted feature-based classifiers (Chatzakou et al., 2017; Davidson et al., 2017; Waseem and Hovy, 2016; MacAvaney et al., 2019), and proposed deep neural network architectures (Zampieri et al., 2019; Gambäck and Sikdar, 2017; Park and Fung, 2017; Badjatiya et al., 2017; Agrawal and Awekar, 2018). However, they might not explore all possible important features for hatespeech detection, ignored pre-trained language model understanding, or proposed uni-directional language models by reading from left to right or right to left.

Recently, the BERT (Bidirectional Encoder Representations from Transformers) model (Devlin et al., 2019) has achieved tremendous success in Natural Language Processing. The key innovation of BERT is in applying the transformer (Vaswani et al., 2017) to language modeling tasks. A BERT model pre-trained on these language modeling tasks forms a good basis for further fine-tuning on supervised tasks such as machine translation and question answering, etc.

Recent work on hatespeech detection (Nikolov and Radivchev, 2019) has applied the BERT model and has shown its prominent results over previous hatespeech classifiers. However, we point out its two limitations in hatespeech detection domain. First, the previous studies (ElSherief et al., $2018 b$,a) have shown that a hateful corpus owns distinguished linguistic/semantic characteristics compared to a non-hateful corpus. For instance, hatespeech sequences are often informal or even in-

\footnotetext{
${ }^{1}$ https://www.nytimes.com/2018/10/31/opinion/caravanhate-speech-bowers-sayoc.html

${ }^{2}$ https://www.washingtonpost.com/nation/2018/11/30/howonline-hate-speech-is-fueling-real-life-violence
} 
tentionally mis-spelled (ElSherief et al., 2018a; Arango et al., 2019), so words in hateful sequences can sit in a long tail when ranking their uniqueness, and a comment can be hateful or non-hateful using the same words (Zhang and Luo, 2019). For example, "dick" in the sentence "Nobody knew dick about what that meant" is non-hateful, but "d1ck" in "You are a weak small-d1cked keyboard warrior" is hateful ${ }^{3}$. Thus, to better understand hateful vocabularies and contexts, it is better to pre-train on a mixture of both hateful and non-hateful corpora. Doing so helps to overcome the limitation of using BERT models pre-trained on non-hateful corpora like English Wikipedia and BookCorpus. Second, even the smallest pre-trained BERT "base" model contains $110 \mathrm{M}$ parameters. It takes a lot of computational resources to pre-train, fine-tune, and serve. Some recent efforts aim to reduce the complexity of BERT model with the knowledge distillation technique such as DistillBert (Sanh et al., 2019) and TinyBert (Jiao et al., 2019). In these methods, a pre-trained BERT-alike model is used as a teacher model, and a student (smaller) model (i.e. TinyBERT, DistilBERT, .etc) is trained to produce similar output to that of the teacher model. Unfortunately, while their complexity is reduced, the performance is also degraded in NLP tasks compared to BERT. Another direction is to use cross-layer parameter sharing, such as ALBERT (Lan et al., 2020). However, ALBERT's computational time is similar to BERT, since the number of layers remains the same as BERT; likewise, its inference is equally expensive.

Based on the above observation and analysis, we aim to investigate whether it is possible to achieve a better hatespeech prediction performance than state-of-the-art machine learning classifiers, including classifiers based on publicly available BERT model, while significantly reducing the number of parameters compared with the BERT model. By doing so, we believe that performing pre-training tasks from the ground up and on a hatespeechrelated corpus would allow the model to understand hatespeech patterns better and enhance the predictive results. However, while language model pretraining tasks require a large scale corpus size,

\footnotetext{
${ }^{3}$ It is important to note that this paper contains hate speech examples, which may be offensive to some readers. They do not represent the views of the authors. We tried to make a balance between showing less number of hate speech examples and illustrating the challenges in real-world applications.
}

available hatespeech datasets are normally small: only $16 \mathrm{~K} \sim 115 \mathrm{~K}$ annotated comments (Waseem and Hovy, 2016; Wulczyn et al., 2017). Thus, we introduce a large annotated hatespeech dataset with 1.4M comments extracted from Yahoo News and Yahoo Finance. To reduce the complexity, we reduce the number of layers and hidden size, and propose Quaternion-based Factorization mechanisms in BERT architecture. To further improve the model effectiveness and robustness, we introduce a multi-source ensemble-head fine-tuning architecture, as well as a target-based adversarial training.

The major contributions of our work are:

- We reduce the number of parameters in BERT considerably, and consequently the training/inferencing time and memory, while achieving better performance compared to the much larger BERT models, and other state-of-the-art hatespeech detection methods.

- We pre-train from the ground up a hateful language model with our proposed Quaternion Factorization methods on a large-scale hatespeech dataset, which gives better performance than fine tuning a pretrained BERT model.

- We propose a flexible classification net with multi-sources and multi-heads, building on top of the learned sequence representations to further enhance our model's predictive capability.

- We utilize adversarial training with a proposed fine-grained and adaptive noise magnitude to improve our model's performance.

\section{Related Work}

Some of the earlier works in hatespeech detection have applied a variety of classical machine learning algorithms (Chatzakou et al., 2017; Davidson et al., 2017; Waseem and Hovy, 2016; MacAvaney et al., 2019). Their intuition is to do feature engineering (i.e. manually generate features), then apply classification methods such as SVM, Random Forest, and Logistic Regression. The features are mostly TermFrequency Inverse-Document-Frequency scores or Bag-of-Words vectors, and can be combined with additional features extracted from the user account's meta information and network structure (i.e., followers, followees, etc). Those methods are suboptimal as they mainly rely on the quality and quantity of the human-crafted features.

Recent works have used deep neural network architectures for hatespeech detection (Zampieri 
et al., 2019; Mou et al., 2020) such as CNN (Gambäck and Sikdar, 2017; Park and Fung, 2017), RNN (i.e. LSTM and GRU) (Badjatiya et al., 2017; Agrawal and Awekar, 2018), combining CNN with RNN (Zhang et al., 2018), or fine tuning a pretrained language models (Indurthi et al., 2019).

Another direction focuses on the testing generalization of the current hatespeech classifiers (Agrawal and Awekar, 2018; Dadvar and Eckert, 2018; Gröndahl et al., 2018), where those methods are tested in other datasets and domains such as Twitter data (Waseem and Hovy, 2016), Wikipedia data (Wulczyn et al., 2017), Formspring data (Reynolds et al., 2011), and YouTube comment data (Dadvar et al., 2014).

Unlike previous works, we pre-train a hateful language model, then build a multi-source multihead hatespeech classifier with regularized adversarial training to enhance the model's performance.

\section{Problem Definition}

Given an input text sequence $s=\left[w_{1}, w_{2}, \ldots, w_{n}\right]$ where $\left\{w_{1}, w_{2}, . ., w_{n}\right\}$ are words and $n=|s|$ is the maximum length of the input sequence $s$. The hatespeech classification task aims to build a mapping function $f: s=\left[w_{1}, w_{2}, \ldots, w_{n}\right] \longrightarrow \mathcal{R} \in[0,1]$, where $f$ inputs $s$ and returns a probability score $P(y=1 \mid s) \in[0,1]$, indicating how likely $s$ is classified as hatespeech. In this paper, we approximate $f$ by a deep neural classifier, where we first pretrain $f$ with unsupervised language modeling tasks to enhance its language understanding. Then, we train $f$ with the hatespeech classification task to produce $P(y=1 \mid s)$.

\section{Our approach - HABERTOR}

\subsection{Tokenization}

BERT model relies on WordPiece (WP) (Wu et al., 2016), a Google's internal code that breaks down each word into common sub-word units ("wordpieces"). These sub-words are like character ngrams, except that they are automatically chosen to ensure that each of these sub-words is frequently observed in the input corpus. WP improves handling of rare words, such as intentionally misspelled abusive words, without the need for a huge vocabulary. A comparable implementation that is open sourced is SentencePiece (SP) (Kudo and Richardson, 2018). Like WP, the vocab size is predetermined. Both WP and SP are unsupervised learning models. Since WP is not released in pub- lic, we train a SP model using our training data, then use it to tokenize input texts.

\subsection{Parameter Reduction with Quaternion Factorization}

Denote $\mathrm{V}$ the vocab size, E the embedding size, $\mathrm{H}$ the hidden size, $\mathrm{L}$ the number of layers, and $\mathrm{F}$ the feed-forward/filter size. In BERT, $\mathrm{F}=4 \mathrm{H}, \mathrm{E}$ $=\mathrm{H}$, and the number of attention heads is $H / 64$. Encoding the vocabs takes VH parameters. Each BERT layer contains three parts: (i) attention, (ii) filtering/feedforward, and (iii) output. Each of the three parts has $4 \mathrm{H}^{2}$ parameters. Thus, a BERT layer has $12 \mathrm{H}^{2}$ parameters and a BERT-base setting with 12 layers has $\mathrm{VH}+144 \mathrm{H}^{2}$ parameters. Please refer to Section A.3 in the Appendix for details.

Recently, Quaternion representations have shown its benefits over Euclidean representations in many neural designs (Parcollet et al., 2019; Tay et al., 2019): (i) a Quaternion number consists of a real component and three imaginary components, encouraging a richer extent of expressiveness; and (ii) a Quaternion transformation reduces $75 \%$ parameters compared to the traditional Euclidean transformation because of the weight sharing using the Hamilton product. Hence, we propose Quaternion fatorization strategies to significantly reduce the model's parameters as follows:

Vocab Factorization (VF): Inspired by Lan et al. (2020), we encode V vocabs using Quaternion representations with an embedding size $\mathrm{E} \ll \mathrm{H}$. Then, we apply a Quaternion transformation to transform $\mathrm{E}$ back to $\mathrm{H}$, and concatenate all four parts of a Quaternion to form a regular Euclidean embedding. This leads to a total of VE $+\mathrm{EH} / 4$ parameters, compared to VE + EH in ALBERT.

Attention Factorization (AF): If the input sequences have length $\mathrm{N}$, the output of the multi-head attention is $\mathrm{N} \times \mathrm{N}$, which does not depend on the hidden size $\mathrm{H}$. Hence, it is unnecessary to produce the attention Query, Key, and Value with the same input hidden size $\mathrm{H}$ and cost $3 \mathrm{H}^{2}$ parameters per a layer. Instead, we produce the attention Query, Key, and Value in size $\mathrm{C} \ll \mathrm{H}$ using linear Quaternion transformations, leading to $3 \mathrm{CH} / 4$ parameters. Feedforward Factorization (FF): Instead of linearly transforming from $\mathrm{H}$ to $4 \mathrm{H}$ (i.e. $4 \mathrm{H}^{2}$ parameters), we apply Quaternion transformations from $\mathrm{H}$ to $\mathrm{I}$, and from $\mathrm{I}$ to $4 \mathrm{H}$, with $\mathrm{I} \ll \mathrm{H}$ is an intermediate size. This leads to a total of $(\mathrm{HI} / 4+\mathrm{IH})$ parameters.

Output Factorization (OF): We also apply 


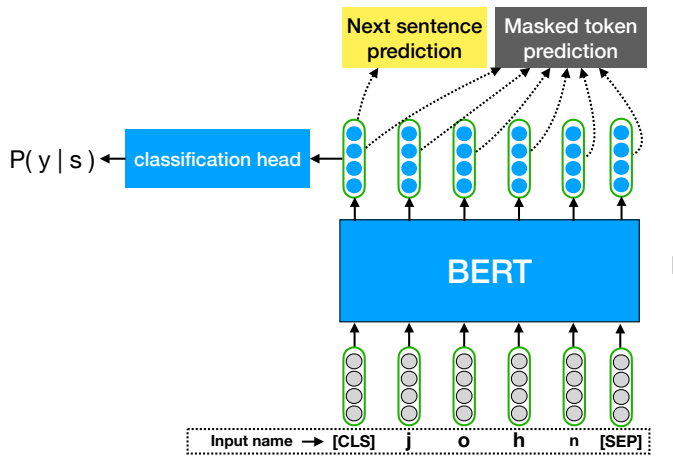

(a) Traditional Fine-tuning BERT.

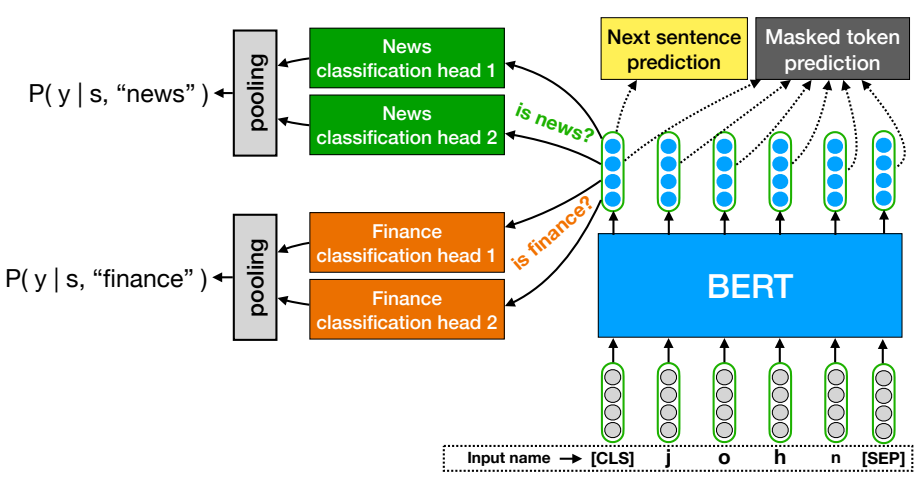

(b) HABERTOR with two sources and ensemble of 2 heads.

Figure 1: Architecture comparison of traditional fine-tuned BERT and HABERTOR multi-source ensemble heads.

Quaternion transformations from $4 \mathrm{H}$ to I, then from I to $\mathrm{H}$. This results in $(\mathrm{HI}+\mathrm{IH} / 4)$ parameters, compared to $4 H^{2}$ in BERT.

When we apply all the above compression techniques together, the total parameters are reduced to $\mathrm{VE}+\mathrm{EH} / 4+\mathrm{L}\left(3 \mathrm{CH} / 4+H^{2}+5 \mathrm{HI} / 2\right)$. Particularly, with BERT-base settings of $\mathrm{V}=32 \mathrm{k}, \mathrm{H}=768, \mathrm{~L}=12$, if we set $\mathrm{E}=128, \mathrm{C}=192$, and $\mathrm{I}=128$, the total of parameters is reduced from $110 \mathrm{M}$ to only $\mathbf{8 . 4 M}$.

\subsection{Pretraining tasks}

Similar to BERT, we pre-train our HABERTOR with two unsupervised learning/language modeling tasks: (i) masked token prediction, and (ii) next sentence prediction. We describe some modifications that we made to the original BERT's implementation as follows:

\subsubsection{Masked token prediction task}

BERT generates only one masked training instance for each input sequence. Instead, inspired by Liu et al. (2019), we generate $\tau$ training instances by randomly sampling with replacement masked positions $\tau$ times. We refer to $\tau$ as a masking factor. Intuitively, this helps the model to learn differently combined patterns of tokens in the same input sequence, and boosts the model's language understanding. This small modification works especially well when we have a smaller pre-training data size, which is often true for a domain-specific task (e.g., hatespeech detection).

\subsubsection{Next sentence prediction task}

In BERT, the two input sentences are already paired and prepared in advanced. In our case, we have to preprocess input text sequences to prepare paired sentences for the next sentence prediction task. We conduct the following preprocessing steps:

Step 1: We train an unsupervised sentence tokenizer from nltk library. Then we use the trained sentence tokenizer to tokenize each input text sequence into (splitted) sentences.

Step 2: In BERT, 50\% of the chance two consecutive sentences are paired as next, and $50 \%$ of the chance two non-consecutive sentences are paired as not next. In our case, our input text sequences can be broken into one, two, three, or more sentences. For input text sequences that consist of only one tokenized sentence, the only choice is to pair with another random sentence to generate a not next example. By following our 50-50 rule described in the Appendix, we ensure generating an equal number of next and not next examples.

\subsection{Training the hatespeech prediction task}

For hatespeech prediction task, we propose a multisource multi-head HABERTOR classifier. The architecture comparison of the traditional fine-tuning BERT and our proposal is shown in Figure 1. We note two main differences in our design as follows.

First, as shown in Figure 1b, our HABERTOR has separated classification heads/nets for different input sequences of different sources but with a shared language understanding knowledge. Intuitively, instead of measuring the same probabilities $P(y \mid s)$ for all input sequences, it injects additional prior source knowledge of the input sequences to measure $P(y \mid s$, "news") or $P(y \mid s$, "finance").

Second, in addition to multi-source, HABERTOR with an ensemble of $h$ heads provides even more capabilities to model data variance. For each input source, we employ ensemble of several classification heads (i.e. two classification heads for each source in the Figure 1b) and use a pooling layer on top to aggregate results from those classification heads. We use three pooling functions: $\min$, max, mean. min pooling indicates that HABERTOR classifies an input comment as a hateful one if all of the heads classify it as hatespeech, which put a more 
stringent requirement on classifying hatespeech. On the other hand, HABERTOR will predict an input comment as a normal comment if at least one of the heads recognizes the input comment as a normal one, which is less strict. Similarly, using max pooling will put more restriction on declaring comments as normal, and less restriction on declaring hatespeech. Finally, mean pooling considers the average voting from all heads.

Note that our design generalizes the traditional fine-tuning BERT architecture when $h=1$ and the two classification nets share the same weights. Thus, HABERTOR is more flexible than the conventional fine-tuning BERT. Also, HABERTOR can be extended trivially to problems that have $q$ sources, with $h$ separated classification heads for $q$ different sources. When predicting input sequences from new sources, HABERTOR averages the scores from all separated classification nets.

\subsection{Parameter Estimation}

Estimating parameters in the pretraining tasks in our model is similar to BERT, and we leave the details in the Appendix due to space limitation.

For hatespeech prediction task, we use the transformed embedding vector of the [CLS] token as a summarized embedding vector for the whole input sequence. Let $S$ be a collection of sequences $s_{i}$. Note that $s_{i}$ is a normal sequence, not corrupted or concatenated with another input sequence. Given that $\boldsymbol{y}_{i}$ is the supervised ground truth label for the input sequence, and $\hat{\boldsymbol{y}}_{i}=P\left(\boldsymbol{y}_{i} \mid s_{i}\right.$, "news") (Figure $1 \mathrm{~b}, 1 \mathrm{~b}$ ) where $s_{i}$ is a news input sequence, or $\hat{\boldsymbol{y}}_{i}=P\left(\boldsymbol{y}_{i} \mid s_{i}\right.$,"finance" $)$ when $s_{i}$ is a finance input sequence. The hateful prediction task aims to minimize the following binary cross entropy loss:

$$
\mathcal{L}_{h s}=\underset{\theta}{\operatorname{argmin}}-\sum_{i=1}^{|\boldsymbol{S}|} \boldsymbol{y}_{i} \log \left(\hat{\boldsymbol{y}}_{i}\right)+\left(1-\boldsymbol{y}_{i}\right) \log \left(1-\hat{\boldsymbol{y}}_{i}\right)
$$

Regularize with adversarial training: To make our model more robust to perturbations of the input embeddings, we further regularize our model with adversarial training. There exist several stateof-the-art target-based adversarial attacks such us Fast Gradient Method (FGM) (Miyato et al., 2017), Basic Iterative Method (Kurakin et al., 2016), and Carlini L2 attack (Carlini and Wagner, 2017). We use the $F G M$ method as it is effective and efficient according to our experiments.

In $F G M$, the noise magnitude is a scalar value and is a manual input hyper-parameter. This is suboptimal, as different adversarial directions of differ- ent dimensions are scaled similarly, plus, manually tuning the noise magnitude is expensive and not optimal. Hence, we propose to extend $F G M$ with a learnable and fine-grained noise magnitude, where the noise magnitude is parameterized by a learnable vector, providing different scales for different adversarial dimensions. Moreover, the running time of our proposal compared to $F G M$ is similar.

The basic idea of the adversarial training is to add a small perturbation noise $\delta$ on each of the token embeddings that makes the model misclassify hateful comments as normal comments, and vice versa. Given the input sequence $s_{i}=$ $\left[w_{1}^{(i)}, w_{2}^{(i)}, \ldots, w_{u}^{(i)}\right]$ with ground truth label $y_{i}$, let $\tilde{y}_{i}$ be the adversarial target class of $s_{i}$ such that $\tilde{y}_{i} \neq y_{i}$. In the hatespeech detection domain, our model is a binary classifier. Hence, when $y_{i}=1$ ( $s_{i}$ is a hateful comment), $\tilde{y}_{i}=0$ and vice versa. Then, the perturbation noise $\delta$ is learned by minimizing the following cost function:

$$
\mathcal{L}_{a d v}=\underset{\delta, \delta \in[a, b]}{\operatorname{argmin}}-\sum_{i=1}^{|\boldsymbol{S}|} \log P\left(\tilde{\boldsymbol{y}}_{\boldsymbol{i}} \mid s_{i}+\delta_{i} ; \hat{\theta}\right)
$$

Note that in Eq. (1), $\delta$ is constrained to be less than a predefined noise magnitude scalar in the traditional FGM method. In our proposal, $\delta$ is constrained within a range $[a, b]$ (i.e. $\min (\delta) \geq \mathrm{a} \wedge$ $\max (\delta) \leq \mathrm{b})$. Solving Eq. (1) is expensive and not easy, especially with complicated deep neural networks. Thus, we approximate each perturbation noise $\delta_{i}$ for each input sequence $s_{i}$ by linearizing partial $\operatorname{loss}-\log P\left(\tilde{\boldsymbol{y}}_{\boldsymbol{i}} \mid s_{i}+\delta_{i} ; \hat{\theta}\right)$ around $s_{i}$. Particularly, $\delta_{i}$ is measured by:

$$
\delta_{i}=-\epsilon \times \frac{\nabla_{s_{i}}\left(-\log P\left(\tilde{\boldsymbol{y}}_{\boldsymbol{i}} \mid s_{i} ; \hat{\theta}\right)\right)}{\left\|\nabla_{s_{i}}\left(-\log P\left(\tilde{\boldsymbol{y}}_{\boldsymbol{i}} \mid s_{i} ; \hat{\theta}\right)\right)\right\|_{2}}
$$

In Eq. (2), $\epsilon$ is a learnable vector, with the same dimensional size as $\delta_{i}$. Solving the constraint $\delta_{i} \in[a, b]$ in Eq. (1) becomes restricting $\epsilon \in[a, b]$, which is trivial by projecting $\epsilon$ in $[a, b]$.

Finally, HABERTOR aims to minimize the following cost function:

$$
\mathcal{L}=\mathcal{L}_{h s}+\lambda_{a d v} \mathcal{L}_{a d v}-\lambda_{\epsilon}\|\epsilon\|_{2},
$$

where $\|\epsilon\|_{2}$ is an additional term to force the model to learn robustly as much as possible, and $\lambda_{\epsilon}$ is a hyper-parameter to balance its effect. Note that, we first learn the optimal values of all token embeddings and HABERTOR's parameters before learning adversarial noise $\delta$. Also, regularizing adversarial training only increases the training time, but not the inferencing time since it does not introduce extra parameters for the model during the inference. 
Table 1: Statistics of the three datasets.

\begin{tabular}{lccc}
\hline Statistics/Datasets & Yahoo & Twitter & Wiki \\
\hline Total & $1.4 \mathrm{M}$ & $16 \mathrm{~K}$ & $115 \mathrm{~K}$ \\
\# Hateful & $100 \mathrm{~K}$ & $5 \mathrm{~K}$ & $13 \mathrm{~K}$ \\
$\%$ of hatespeech & $7 \%$ & $31 \%$ & $12 \%$ \\
\hline
\end{tabular}

\section{Empirical Study}

\subsection{Experiment Setting}

Dataset: Our primary dataset was extracted from user comments on Yahoo News and Finance for five years, and consisted of 1,429,138 labeled comments. Among them, 944,391 comments are from Yahoo News and 484,747 comments are from Yahoo Finance. There are 100,652 hateful comments. The $1.4 \mathrm{M}$ labeled data was collected as follows (Nobata et al., 2016): comments that are reported as "abusive" for any reason by users of Yahoo News and Finance are sent to in-house trained raters for review and labeling.

To further validate the generalizability of HABERTOR, we perform transfer-learning experiments on other two publicly available hatespeech datasets: Twitter (Waseem and Hovy, 2016), and Wikipedia (i.e. Wiki) (Wulczyn et al., 2017). The Twitter dataset consists of $16 \mathrm{~K}$ annotated tweets, including 5,054 hateful tweets (i.e., 31\%). The Wiki dataset has $115 \mathrm{~K}$ labeled discussion comments from English Wikipedia talk's page, including 13,590 hateful comments (i.e., 12\%). The statistics of 3 datasets are shown in Table 1.

Train/Dev/Test split: We split the dataset into train/dev/test sets with a ratio $70 \% / 10 \% / 20 \%$. We tune hyper-parameters on the dev set, and report final results on the test set. Considering critical mistakes reported at Arango et al. (2019) when building machine learning models (i.e. extracting features using the entire dataset, including testing data, etc), we generate vocabs, pre-train the two language modeling tasks, and train the hatespeech prediction task using only the training set.

Baselines, our Models and hyper-parameter Settings: We compare our models with 15 state-ofthe-art baselines: Bag of Words (BOW) (Dinakar et al., 2011; Van Hee et al., 2015), NGRAM, CNN (Kim, 2014), VDCNN (Conneau et al., 2017), FastText (Joulin et al., 2016), LSTM (Cho et al., 2014), att-LSTM, RCNN (Lai et al., 2015), att-BiLSTM (Lin et al., 2017), Fermi (best hatespeech detection method as reported in Basile et al. (2019)) (Indurthi et al., 2019), Q-Transformer (Tay et al., 2019), Tiny-BERT (Jiao et al., 2019), DistilBERT-
Table 2: Parameters Comparison between HABERTORVAFOQF vs. other LMs. "-" indicates not available.

\begin{tabular}{lccccc}
\hline \multirow{2}{*}{ Statistics } & HABERTOR & AL- & \multicolumn{3}{c}{ Tiny- } \\
& -VAFOQF & BERT & BERT & BERT & base \\
\hline Layers (L) & $\mathbf{6}$ & 12 & 4 & 6 & 12 \\
Attention heads & $\mathbf{6}$ & 12 & 12 & 12 & 12 \\
Attention size (C) & $\mathbf{1 9 2}$ & - & - & - & - \\
Embedding (E) & $\mathbf{1 2 8}$ & 128 & - & - & - \\
Hidden (H) & $\mathbf{3 8 4}$ & 768 & 312 & 768 & 768 \\
Intermediate size (I) & $\mathbf{1 2 8}$ & - & - & - & - \\
Feedforward size & $\mathbf{1 , 5 3 6}$ & 3072 & 1,200 & 3,072 & 3,072 \\
Vocab (V) & $\mathbf{4 0 k}$ & $30 \mathrm{k}$ & $30 \mathrm{k}$ & $30 \mathrm{k}$ & $30 \mathrm{k}$ \\
\hline Parameters & $\mathbf{7 . 1 M}$ & $12 \mathrm{M}$ & $14.5 \mathrm{M}$ & $65 \mathrm{M}$ & $110 \mathrm{M}$ \\
\hline
\end{tabular}

base (Sanh et al., 2019), ALBERT-base (Lan et al., 2020), and BERT-base (Devlin et al., 2019; Nikolov and Radivchev, 2019). We are aware of other recent language models such as Transformer-XL (Dai et al., 2019), RoBERTa(Liu et al., 2019), DialoGPT (Zhang et al., 2020), to name a few. However, as these models are even heavier than BERT-base, we do not compare with them. The detailed description of the baselines and hyper-parameter settings is described in the Appendix.

Our models: We denote HABERTOR as our model without using any factorization, HABERTOR$V Q F$ as HABERTOR + Vocab Quaternion Factorization, HABERTOR-VAQF as HABERTOR + Vocab + Attention Quaternion Factorization, HABERTOR-VAFQF as HABERTOR + Vocab + Attention + Feedforward Quaternion Factorization, and HABERTOR-VAFOQF as HABERTOR + Vo$\mathrm{cab}+$ Attention + Feedforward + Output Quaternion Factorization.

Measurement: We evaluate models on seven metrics: Area Under the Curve $(A U C)$, Average Precision $(A P)$, False Positive Rate (FPR), False Negative Rate (FNR), F1 score ${ }^{4}$. In real world, for imbalanced datasets, we care more about FPR and FNR. Thus, we report FPR at 5\% of FNR (FPR@ $5 \%$ FNR), meaning we allow 5\% of hateful texts to be misclassified as normal ones, then report FPR at that point. Similarly, we report FNR at $5 \%$ of FPR (FNR@5\%FPR). Except for AUC and AP, the other metrics are reported using an optimal threshold selected by using the development set.

Model size comparison: HABERTOR has $26 \mathrm{M}$ of parameters. HABERTOR-VQF and HABERTOR$V A Q F$ have $16.2 \mathrm{M}$ and $13.4 \mathrm{M}$ of parameters, respectively. HABERTOR-VAFQF and HABERTORVAFOQF have $10.3 \mathrm{M}$ and $7.1 \mathrm{M}$ of parameters, respectively. The size of all five models is much

\footnotetext{
${ }^{4}$ Both AP and F1 account for Precision and Recall so we do not further report Precision and Recall for saving space.
} 
Table 3: Performance of all models that we train on Yahoo train data, test on Yahoo test data and report results on Yahoo News and Yahoo Finance separately. Best baseline is underlined, better results than best baseline are bold.

\begin{tabular}{|c|c|c|c|c|c|c|c|c|c|c|c|c|}
\hline \multirow{2}{*}{ Model } & \multicolumn{2}{|c|}{ Yahoo } & \multicolumn{5}{|c|}{ Yahoo News } & \multicolumn{5}{|c|}{ Yahoo Finance } \\
\hline & AUC & AP & AUC & AP & $\begin{array}{c}\text { FPR@ } \\
5 \% \text { FNR }\end{array}$ & $\begin{array}{l}\text { FNR@ } \\
5 \% \text { FPR }\end{array}$ & $\mathrm{F} 1$ & AUC & AP & $\begin{array}{c}\text { FPR@ } \\
5 \% \text { FNR }\end{array}$ & $\begin{array}{l}\text { FNR@ } \\
5 \% \text { FPR }\end{array}$ & $\mathrm{F} 1$ \\
\hline BOW & 85.91 & 48.35 & 85.07 & 51.37 & 61.13 & 50.53 & 49.01 & 85.83 & 36.80 & 60.97 & 49.43 & 40.15 \\
\hline NGRAM & 84.19 & 42.15 & 83.17 & 45.00 & 63.45 & 57.45 & 43.59 & 84.29 & 31.63 & 63.42 & 53.94 & 35.95 \\
\hline $\mathrm{CNN}$ & 91.21 & 63.03 & 90.64 & 65.64 & 47.50 & 36.23 & 60.61 & 91.20 & 52.30 & 45.59 & 33.96 & 51.93 \\
\hline VDCNN & 88.10 & 58.08 & 87.65 & 60.75 & 60.39 & 41.56 & 56.12 & 88.17 & 48.72 & 62.43 & 38.78 & 50.38 \\
\hline FastText & 91.64 & 60.15 & 90.97 & 63.16 & 41.80 & 38.09 & 58.35 & 92.13 & 47.97 & 37.75 & 34.30 & 49.36 \\
\hline LSTM & 91.83 & 64.17 & 91.14 & 66.59 & 43.81 & 35.09 & 60.96 & 92.38 & 54.44 & 38.26 & 31.45 & 53.36 \\
\hline att-LSTM & 91.83 & 64.39 & 91.10 & 66.77 & 44.24 & 34.86 & 61.37 & 92.43 & 54.79 & 38.32 & 30.75 & 53.79 \\
\hline RCNN & 91.17 & 63.34 & 90.52 & 65.72 & 48.49 & 36.37 & 60.29 & 91.32 & 53.77 & 49.40 & 32.17 & 52.73 \\
\hline att-BiLSTM & 92.52 & 64.17 & 91.93 & 66.82 & 38.07 & 34.68 & 61.54 & 92.93 & 53.97 & 36.05 & 31.14 & 52.58 \\
\hline Fermi & 86.53 & 41.52 & 86.10 & 45.16 & 53.33 & 55.60 & 45.65 & 85.45 & 27.53 & 56.60 & 56.48 & 33.27 \\
\hline Q-Transformer & 92.34 & 64.43 & 91.81 & 67.06 & 39.12 & 34.17 & 61.82 & 92.64 & 54.41 & 37.71 & 29.74 & 53.51 \\
\hline Tiny-BERT & 93.60 & 68.70 & 93.03 & 70.80 & 34.50 & 30.37 & 64.42 & 94.09 & 60.25 & 31.16 & 25.09 & 57.58 \\
\hline DistilBERT & 93.68 & 69.15 & 93.13 & 71.25 & 34.33 & 30.05 & 64.69 & 94.12 & 60.56 & 29.23 & 24.94 & 58.01 \\
\hline ALBERT & 93.50 & 67.99 & 92.93 & 70.28 & 34.56 & 31.15 & 63.82 & 93.94 & 58.73 & 30.12 & 25.87 & 56.37 \\
\hline BERT-base & 94.14 & $\underline{70.05}$ & 93.56 & $\underline{71.65}$ & $\underline{32.15}$ & 28.91 & $\underline{65.30}$ & 94.60 & $\underline{62.34}$ & 29.14 & 22.81 & $\underline{59.72}$ \\
\hline HABERTOR & 94.77 & 72.35 & 94.12 & 73.79 & 29.26 & 27.12 & 67.09 & 95.72 & 65.93 & 22.03 & 18.99 & 62.38 \\
\hline HABERTOR-VQF & 94.70 & 71.82 & 94.00 & 73.25 & 29.50 & 27.79 & 66.57 & 95.81 & 65.20 & 20.78 & 20.08 & 61.60 \\
\hline HABERTOR-VAQF & 94.59 & 71.53 & 93.90 & 73.02 & 29.94 & 27.92 & 66.51 & 95.63 & 64.64 & 23.08 & 20.39 & 60.84 \\
\hline HABERTOR-VAFQF & 94.43 & 70.75 & 93.72 & 72.37 & 31.86 & 28.58 & 65.81 & 95.42 & 63.07 & 22.87 & 21.43 & 60.11 \\
\hline HABERTOR-VAFOQF & 94.18 & 69.92 & 93.51 & 71.63 & 32.47 & 29.26 & 65.35 & 95.00 & 61.99 & 24.95 & 22.81 & 59.50 \\
\hline
\end{tabular}

smaller than BERT-base (i.e. 110M of parameters). The configuration comparison of HABERTORVAFOQF and other pretrained language models is given in Table 2. HABERTOR-VAFOQF has less than 2 times compared to TinyBERT's parameters, less than 9 times compared to Distil-BERT's size, and is equal to 0.59 AlBERT's size.

\subsection{Experimental results}

\subsubsection{Performance comparison}

Table 3 shows the performance of all models on Yahoo dataset. Note that we train on the Yahoo training set that contains both Yahoo News and Finance data, and report results on Yahoo News and Finance separately, and report only AUC and AP on both of them (denoted as column "Yahoo" in Table 3). We see that Fermi worked worst among all models. It is mainly because Fermi transfers the pre-trained embeddings from the USE model to a SVM classifier without further fine-tuning. This limits Fermi's ability to understand domain-specific contexts. Q-Transformer works the best among non-LM baselines, but worse than LM baselines as it is not pretrained. BERT-base performed the best among all baselines. Also, distilled models worked worse than BERT-base due to their compression nature on BERT-base as the teacher model.

Next, we compare the performance of our proposed models against each other. Table 3 shows that our models' performance is decreasing when we compress more components ( $p$-value $<0.05$ un- der the directional Wilcoxon signed-rank test). We reason it is a trade-off between the model size and the model performance as factorizing a component will naturally lose some of its information.

Then, we compared our proposed models with BERT-base - the best baseline. Table 3 shows that except our HABERTOR-VAFOQF, our other proposals outperformed BERT-base, improving by an average of $1.2 \%$ and $1.5 \%$ of F1-score in Yahoo News and Yahoo Finance, respectively ( $p$-value $<$ $0.05)$. Recall that in addition to improving hatespeech detection performance, our models' size is much smaller than BERT-base. For example, HABERTOR saved 84M of parameters from BERTbase, and HABERTOR-VAFQF saved nearly 100M of parameters from BERT-base. Interestingly, even our smallest HABERTOR-VAFOQF model (7.1M of parameters) achieves similar results compared to BERT-base (i.e. the performance difference between them is not significant under the directional Wilcoxon signed-rank test). Those results show the effectiveness of our proposed models against BERT-base, the best baseline, and consolidate the need of pretraining a language model on a hateful corpus for a better hateful language understanding.

\subsubsection{Running time and memory comparison}

Running time: Among LM baselines, TinyBERT is the fastest. Though ALBERT has the smallest number of parameters by adopting the cross-layer weight sharing mechanism, ALBERT has the same 
Table 4: Generalizability of HABERTOR and top baselines. Report AUC, AP, and F1 on each test set.

\begin{tabular}{l|ccl|ccc}
\hline & \multicolumn{3}{|c|}{ Twitter } & \multicolumn{3}{c}{ Wiki } \\
\hline Model & AUC & AP & F1 & AUC & AP & F1 \\
\hline Fermi & 89.03 & 79.23 & 74.52 & 96.59 & 84.26 & 75.51 \\
TinyBERT & 92.23 & 83.88 & 78.33 & 97.10 & 87.64 & 79.70 \\
DistilBERT & 92.13 & 80.21 & 77.89 & 97.23 & 88.16 & 80.21 \\
ALBERT & 92.55 & 86.51 & 78.76 & 97.66 & 88.91 & 80.66 \\
BERT & 93.21 & $\underline{86.67}$ & $\underline{79.68}$ & $\underline{97.75}$ & $\underline{89.23}$ & $\underline{80.73}$ \\
\hline HABERTOR & $\mathbf{9 3 . 5 2}$ & $\mathbf{8 8 . 5 7}$ & $\mathbf{8 1 . 2 2}$ & 97.46 & 88.65 & $\mathbf{8 0 . 8 1}$ \\
HABERTOR-VQF & $\mathbf{9 3 . 9 4}$ & $\mathbf{8 8 . 4 5}$ & $\mathbf{8 1 . 2 1}$ & 97.40 & 88.64 & 80.66 \\
HABERTOR-VAQF & $\mathbf{9 3 . 5 7}$ & $\mathbf{8 7 . 6 6}$ & $\mathbf{8 0 . 2 3}$ & 97.45 & 88.61 & 80.63 \\
HABERTOR-VAFQF & $\mathbf{9 3 . 5 1}$ & $\mathbf{8 7 . 3 8}$ & $\mathbf{8 0 . 1 6}$ & 97.37 & 88.21 & 80.23 \\
HABERTOR-VAFOQF & $\mathbf{9 3 . 4 9}$ & $\mathbf{8 7 . 1 4}$ & $\mathbf{8 0 . 0 6}$ & 97.23 & 87.94 & 79.61 \\
\hline
\end{tabular}

number of layers as BERT-base, leading to a similar computational expense as BERT-base.

Our HABERTOR-VQF and HABERTOR-VAQF have a very similar parameter size with TinyBERT and their train/inference time are similar. Interestingly, even though HABERTOR has $26 \mathrm{M}$ of parameters, its runtime is also competitive with Tiny$B E R T$. This is because among $26 \mathrm{M}$ of parameters in HABERTOR, $15.4 \mathrm{M}$ of its total parameters are for encoding $40 \mathrm{k}$ vocabs, which are not computational parameters and are only updated sparsely during training. HABERTOR-VAFQF and HABERTOR$V A F O Q F$ significantly reduce the number of parameters compared to TinyBERT, leading to a speedup during training and inference phases. Especially, our experiments on $4 \mathrm{~K} 80$ GPUs with a batch size of 128 shows that HABERTOR-VAFOQF is 1.6 times faster than TinyBERT.

Memory consumption: Our experiments with a batch size of 128 on 4 K80 GPUs show that among LM baselines, TinyBERT and ALBERT are the most lightweight models, consuming 13GB of GPU memory. Compared to TinyBERT and ALBERT, HABERTOR takes an additional 4GB of GPU memory, while HABERTOR-VQF, HABERTOR-VAQF hold a similar memory consumption, HABERTOR-VAFQF and HABERTOR$V A F O Q F$ reduces $1 \sim 3$ GB of GPU memory.

Compared to BERT-base: In general, HABERTOR is $4 \sim 5$ times faster, and 3.1 times GPU memory usage smaller than BERT-base. Our most lightweight model HABERTOR-VAFOQF even reduces 3.6 times GPU memory usage, while remains as effective as BERT-base. The memory saving in our models also indicates that we could increase the batch size to perform inference even faster.

\subsubsection{Generalizability analysis}

We perform hatespeech Language Model transfer learning on other hateful Twitter and Wiki datasets to understand our models' generalizability. We use
Table 5: Comparison of the traditional FGM with a fixed and scalar noise magnitude, compared to the FGM with our proposed fine-grained and adaptive noise magnitude. Better results are in bold.

\begin{tabular}{c|c|ccc|ccc}
\hline & & \multicolumn{3}{|c|}{ Twitter } & \multicolumn{3}{c}{ Wiki } \\
\hline Model & Type & AUC & AP & F1 & AUC & AP & F1 \\
\hline \multirow{2}{*}{ HABERTOR } & traditional & 93.54 & 87.88 & 79.84 & 97.50 & 88.14 & 80.13 \\
\cline { 2 - 8 } & ours & 93.52 & $\mathbf{8 8 . 5 7}$ & $\mathbf{8 1 . 2 2}$ & 97.46 & $\mathbf{8 8 . 6 5}$ & $\mathbf{8 0 . 8 1}$ \\
\hline \hline \multirow{2}{*}{$\begin{array}{c}\text { HABERTOR- } \\
\text { VQF }\end{array}$} & traditional & 93.62 & 88.09 & 80.26 & 97.44 & 88.19 & 80.11 \\
\cline { 2 - 8 } & ours & 93.94 & $\mathbf{8 8 . 4 5}$ & $\mathbf{8 1 . 2 1}$ & 97.40 & $\mathbf{8 8 . 6 4}$ & $\mathbf{8 0 . 6 6}$ \\
\hline \hline \multirow{2}{*}{$\begin{array}{c}\text { HABERTOR- } \\
\text { VAQF }\end{array}$} & traditional & 93.03 & 86.77 & 79.56 & 97.44 & 88.15 & 80.16 \\
\cline { 2 - 8 } & ours & 93.57 & $\mathbf{8 7 . 6 6}$ & $\mathbf{8 0 . 2 3}$ & 97.45 & $\mathbf{8 8 . 6 1}$ & $\mathbf{8 0 . 6 3}$ \\
\hline \hline \multirow{2}{*}{$\begin{array}{c}\text { HABERTOR- } \\
\text { VAFQF }\end{array}$} & traditional & 92.89 & 86.42 & 79.64 & 97.42 & 88.08 & 79.71 \\
\cline { 2 - 8 } & ours & 93.51 & $\mathbf{8 7 . 3 8}$ & $\mathbf{8 0 . 1 6}$ & 97.37 & $\mathbf{8 8 . 2 1}$ & $\mathbf{8 0 . 2 3}$ \\
\hline \hline \multirow{2}{*}{$\begin{array}{c}\text { HABERTOR- } \\
\text { VAFOQF }\end{array}$} & traditional & 93.08 & 86.67 & 79.33 & 97.28 & 87.40 & 79.19 \\
\cline { 2 - 8 } & ours & 93.49 & $\mathbf{8 7 . 1 4}$ & $\mathbf{8 0 . 0 6}$ & 97.23 & $\mathbf{8 7 . 9 4}$ & $\mathbf{7 9 . 6 1}$ \\
\hline
\end{tabular}

our models' pre-trained language model checkpoint learned from Yahoo hateful datasets, and fine tune them on Twitter/Wiki datasets. Note that the finetuned training also includes regularized adversarial training for best performance. Next, we compare the performance of our models with Fermi and four LM baselines - best baselines reported in Table 3 .

Table 4 shows that BERT-base performed best compared to other fine-tuned LMs, which is consistent with our reported results on Yahoo datasets in Table 3. When comparing with BERT-base's performance (i.e. best baseline) on the Twitter dataset, all our models outperformed BERT-base. On Wiki dataset, interestingly, our models work very competitively with BERT-base, and achieve similar F1score results. Recall that BERT-base has a major advantage of pre-training on 2,500M Wiki words, thus potentially understands Wiki language styles and contexts better. In contrast, HABERTOR and its four factorized versions are pre-trained on 33M words from Yahoo hatespeech dataset. As shown in the ablation study (refer to AS2 in Section A.6 of the Appendix), a larger pre-training data size leads to better language understanding and a higher hatespeech prediction performance. Hence, if we acquire larger pre-training data with more hateful representatives, our model's performance can be further boosted. All of those results show that our models generalize well on other hatespeech datasets compared with BERT-base, with significant model complexity reduction.

\subsubsection{Ablation study}

Effectiveness of the adversarial attacking method FGM with our fined-grained and adaptive noise magnitude: To show the effectiveness of the FGM attacking method with our proposed fine-grained and adaptive noise magnitude, we 


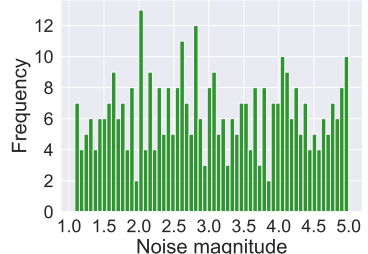

(a) Twitter

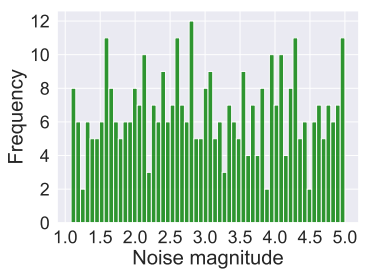

(b) Wiki.
Figure 2: Histogram of the learned noise magnitude when performing Language Model transfer learning of HABERTOR on (a) Twitter, and (b) Wiki datasets.

compare the performance of HABERTOR and its four factorized versions when (i) using a fixed and scalar noise magnitude as in the traditional FGM method, and (ii) using a fine-grained and adaptive noise magnitude in our proposal. We evaluate the results by performing the Language Model transfer learning on Twitter and Wiki datasets and present results in Table 5. Note that, the noise magnitude range is set in $[1,5]$ for both two cases (i) and (ii) for a fair comparison, and we manually search the optimal value of the noise magnitude in the traditional FGM method using the development set in each dataset. We observe that in all our five models, learning with our modified FGM produces better results compared to learning with a traditional FGM, confirming the effectiveness of our proposed finegrained and adaptive noise magnitude.

We also plot the histogram of the learned noise magnitude of HABERTOR on Twitter and Wiki datasets. Figure 2 shows that different embedding dimensions are assigned with different learned noise magnitude, showing the need of our proposed fine-grained and adaptive noise magnitude, that automatically assigns different noise scales for different embedding dimensions.

Additional Ablation study: We conduct several ablation studies to understand HABERTOR's sensitivity. Due to space limitation, we summarize the key findings as follows, and leave detailed information and additional study results in the Appendix: (i) A large masking factor in HABERTOR is helpful to improve its performance; (ii) Pretraining with a larger hatespeech dataset or a more fine-grained pretraining can improve the hatespeech prediction performance; and (iii) Our fine-tuning architecture with multi-source and ensemble of classification heads helps improve the performance.

\subsubsection{Further application discussion}

Our proposals were designed for the hatespeech detection task, but in an extent, they can be applied for other text classification tasks. To illus-
Table 6: Application of our models on the sentiment classification task using Amazon Prime Pantry reviews.

\begin{tabular}{l|ccc}
\hline Model & AUC & AP & F1 \\
\hline ALBERT-base & 98.77 & 99.77 & 97.95 \\
BERT-base & 99.16 & 99.84 & 98.42 \\
\hline HABERTOR & 99.10 & 99.83 & 98.39 \\
HABERTOR+VQF & 99.09 & 99.83 & 98.27 \\
HABERTOR+VAQF & 98.90 & 99.80 & 98.07 \\
HABERTOR+VAFQF & 98.87 & 99.79 & 98.05 \\
HABERTOR+VAFOQF & 98.61 & 99.75 & 97.78 \\
\hline
\end{tabular}

trate the point, we experiment our models (i.e. all our pretraining and fine-tuning designs) on a sentiment classification task. Particularly, we used 471k Amazon-Prime-Pantry reviews (McAuley et al., 2015), which is selected due to its reasonable size for fast pretraining, fine-tuning and result attainment. After some preprocessings (i.e. duplicated reviews removal, convert the reviews with rating scores $\geq 4$ as positive, rating $\leq 2$ as negative, and no neutral class for easy illustration), we obtained $301 \mathrm{k}$ reviews and splited into $210 \mathrm{k}-$ training/30k-development/60k-testing with a ratio $70 / 10 / 20$. Next, we pretrained our models on $210 \mathrm{k}$ training reviews which contain $5.06 \mathrm{M}$ of words. Then, we fine-tuned our models on the 210k training reviews, selected a classification threshold on the 30k development reviews, and report AUC, $\mathrm{AP}$, and $\mathrm{F} 1$ on the $60 \mathrm{k}$ testing reviews. We compare the performance of our models with fine-tuned BERT-base and ALBERT-base - two best baselines. We observe that though pretraining on only $5.06 \mathrm{M}$ words of 210k training reviews, HABERTOR performs very similarly to BERT-base, while improving over ALBERT-base. Except HABERTORVAFOQF with a little bit smaller F1-score compared to ALBERT-base, our other three compressed models worked better than ALBERT-base, showing the effectiveness of our proposals.

\section{Conclusion}

In this paper, we presented the HABERTOR model for detecting hatespeech. HABERTOR understands the language of the hatespeech datasets better, is 4-5 times faster, uses less than $1 / 3$ of the memory, and has a better performance in hatespeech classification. Overall, HABERTOR outperforms 15 state-of-the-art hatespeech classifiers and generalizes well to unseen hatespeech datasets, verifying not only its efficiency but also its effectiveness.

\section{Acknowledgments}

This work was supported in part by NSF grant CNS-1755536. 


\section{References}

Sweta Agrawal and Amit Awekar. 2018. Deep learning for detecting cyberbullying across multiple social media platforms. In ECIR, pages 141-153.

Aymé Arango, Jorge Pérez, and Barbara Poblete. 2019. Hate speech detection is not as easy as you may think: A closer look at model validation. In SIGIR, pages $45-54$.

Pinkesh Badjatiya, Shashank Gupta, Manish Gupta, and Vasudeva Varma. 2017. Deep learning for hate speech detection in tweets. In $W W W$ Companion, pages 759-760.

Barna. 2019. U.S. adults believe hate speech has increased - mainly online. research releases in culture \& media. https://www.barna.com/ research/hate-speech-increased/.

Valerio Basile, Cristina Bosco, Elisabetta Fersini, Debora Nozza, Viviana Patti, Francisco Manuel Rangel Pardo, Paolo Rosso, and Manuela Sanguinetti. 2019. SemEval-2019 task 5: Multilingual detection of hate speech against immigrants and women in twitter. In SemEval, pages 54-63.

Nicholas Carlini and David Wagner. 2017. Towards evaluating the robustness of neural networks. In $S P$, pages 39-57.

Daniel Cer, Yinfei Yang, Sheng-yi Kong, Nan Hua, Nicole Limtiaco, Rhomni St John, Noah Constant, Mario Guajardo-Cespedes, Steve Yuan, Chris Tar, et al. 2018. Universal sentence encoder for english. In EMNLP: System Demonstrations, pages 169-174.

Despoina Chatzakou, Nicolas Kourtellis, Jeremy Blackburn, Emiliano De Cristofaro, Gianluca Stringhini, and Athena Vakali. 2017. Mean birds: Detecting aggression and bullying on twitter. In WebSci, pages 13-22.

Kyunghyun Cho, Bart Van Merriënboer, Caglar Gulcehre, Dzmitry Bahdanau, Fethi Bougares, Holger Schwenk, and Yoshua Bengio. 2014. Learning phrase representations using rnn encoder-decoder for statistical machine translation. In EMNLP.

Kevin Clark, Urvashi Khandelwal, Omer Levy, and Christopher D Manning. 2019. What does bert look at? an analysis of bert's attention. arXiv preprint arXiv:1906.04341.

Alexis Conneau, Holger Schwenk, Loïc Barrault, and Yann Lecun. 2017. Very deep convolutional networks for text classification. In EACL, pages 11071116.

Maral Dadvar and Kai Eckert. 2018. Cyberbullying detection in social networks using deep learning based models; a reproducibility study. arXiv preprint arXiv:1812.08046.
Maral Dadvar, Dolf Trieschnigg, and Franciska de Jong. 2014. Experts and machines against bullies: A hybrid approach to detect cyberbullies. In CCAI, pages 275-281.

Zihang Dai, Zhilin Yang, Yiming Yang, William W Cohen, Jaime Carbonell, Quoc V Le, and Ruslan Salakhutdinov. 2019. Transformer-xl: Attentive language models beyond a fixed-length context. In $A C L$.

Thomas Davidson, Dana Warmsley, Michael Macy, and Ingmar Weber. 2017. Automated hate speech detection and the problem of offensive language. In ICWSM.

Jacob Devlin, Ming-Wei Chang, Kenton Lee, and Kristina Toutanova. 2019. Bert: Pre-training of deep bidirectional transformers for language understanding. In NAACL-HLT.

Karthik Dinakar, Roi Reichart, and Henry Lieberman. 2011. Modeling the detection of textual cyberbullying. In ICWSM.

Mai ElSherief, Vivek Kulkarni, Dana Nguyen, William Yang Wang, and Elizabeth Belding. 2018a. Hate lingo: A target-based linguistic analysis of hate speech in social media. In ICWSM.

Mai ElSherief, Shirin Nilizadeh, Dana Nguyen, Giovanni Vigna, and Elizabeth Belding. 2018b. Peer to peer hate: Hate speech instigators and their targets. In ICWSM.

Björn Gambäck and Utpal Kumar Sikdar. 2017. Using convolutional neural networks to classify hatespeech. In ACL workshop on abusive language online, pages 85-90.

Tommi Gröndahl, Luca Pajola, Mika Juuti, Mauro Conti, and N Asokan. 2018. All you need is: Evading hate speech detection. In AISEC, pages 2-12.

Vijayasaradhi Indurthi, Bakhtiyar Syed, Manish Shrivastava, Nikhil Chakravartula, Manish Gupta, and Vasudeva Varma. 2019. FERMI at SemEval-2019 task 5: Using sentence embeddings to identify hate speech against immigrants and women in twitter. In SemEval, pages 70-74. ACL.

Xiaoqi Jiao, Yichun Yin, Lifeng Shang, Xin Jiang, Xiao Chen, Linlin Li, Fang Wang, and Qun Liu. 2019. Tinybert: Distilling bert for natural language understanding. arXiv preprint arXiv:1909.10351.

Armand Joulin, Edouard Grave, Piotr Bojanowski, Matthijs Douze, Hérve Jégou, and Tomas Mikolov. 2016. Fasttext. zip: Compressing text classification models. arXiv preprint arXiv: 1612.03651.

Yoon Kim. 2014. Convolutional neural networks for sentence classification. In EMNLP.

Diederik P Kingma and Jimmy Ba. 2014. Adam: A method for stochastic optimization. arXiv preprint arXiv:1412.6980. 
Taku Kudo and John Richardson. 2018. SentencePiece: A simple and language independent subword tokenizer and detokenizer for neural text processing. In EMNLP: System Demonstrations.

Alexey Kurakin, Ian Goodfellow, and Samy Bengio. 2016. Adversarial examples in the physical world In Adversarial examples in the physical world.

Siwei Lai, Liheng Xu, Kang Liu, and Jun Zhao. 2015. Recurrent convolutional neural networks for text classification. In $A A A I$.

Zhenzhong Lan, Mingda Chen, Sebastian Goodman, Kevin Gimpel, Piyush Sharma, and Radu Soricut. 2020. Albert: A lite bert for self-supervised learning of language representations. In ICLR.

Zhouhan Lin, Minwei Feng, Cicero Nogueira dos Santos, Mo Yu, Bing Xiang, Bowen Zhou, and Yoshua Bengio. 2017. A structured self-attentive sentence embedding. In ICLR.

Yinhan Liu, Myle Ott, Naman Goyal, Jingfei Du, Mandar Joshi, Danqi Chen, Omer Levy, Mike Lewis, Luke Zettlemoyer, and Veselin Stoyanov. 2019. Roberta: A robustly optimized bert pretraining approach. arXiv preprint arXiv:1907.11692.

Sean MacAvaney, Hao-Ren Yao, Eugene Yang, Katina Russell, Nazli Goharian, and Ophir Frieder. 2019. Hate speech detection: Challenges and solutions. PloS one, 14(8):e0221152.

Julian McAuley, Christopher Targett, Qinfeng Shi, and Anton Van Den Hengel. 2015. Image-based recommendations on styles and substitutes. In SIGIR, pages 43-52.

Takeru Miyato, Andrew M Dai, and Ian Goodfellow. 2017. Adversarial training methods for semisupervised text classification. In ICLR.

Guanyi Mou, Pengyi Ye, and Kyumin Lee. 2020 Swe2: Subword enriched and significant word emphasized framework for hate speech detection. In CIKM.

Alex Nikolov and Victor Radivchev. 2019. Nikolovradivchev at semeval-2019 task 6: Offensive tweet classification with bert and ensembles. In $A C L S e$ mEval, pages 691-695.

Chikashi Nobata, Joel Tetreault, Achint Thomas, Yashar Mehdad, and Yi Chang. 2016. Abusive language detection in online user content. In $W W W$.

Titouan Parcollet, Mirco Ravanelli, Mohamed Morchid, Georges Linarès, Chiheb Trabelsi, Renato De Mori, and Yoshua Bengio. 2019. Quaternion recurrent neural networks. In International Conference on Learning Representations.

Ji Ho Park and Pascale Fung. 2017. One-step and twostep classification for abusive language detection on twitter. In ACL Workshop on Abusive Language Online.
Jeffrey Pennington, Richard Socher, and Christopher D. Manning. 2014. Glove: Global vectors for word representation. In In EMNLP.

Kelly Reynolds, April Kontostathis, and Lynne Edwards. 2011. Using machine learning to detect cyberbullying. In ICMLA, volume 2, pages 241-244.

Victor Sanh, Lysandre Debut, Julien Chaumond, and Thomas Wolf. 2019. Distilbert, a distilled version of bert: smaller, faster, cheaper and lighter. arXiv preprint arXiv:1910.01108.

Yi Tay, Aston Zhang, Anh Tuan Luu, Jinfeng Rao, Shuai Zhang, Shuohang Wang, Jie Fu, and Siu Cheung Hui. 2019. Lightweight and efficient neural natural language processing with quaternion networks. In $A C L$, pages 1494-1503.

Cynthia Van Hee, Els Lefever, Ben Verhoeven, Julie Mennes, Bart Desmet, Guy De Pauw, Walter Daelemans, and Véronique Hoste. 2015. Automatic detection and prevention of cyberbullying. In HUSO, pages $13-18$.

Ashish Vaswani, Noam Shazeer, Niki Parmar, Jakob Uszkoreit, Llion Jones, Aidan N Gomez, Łukasz Kaiser, and Illia Polosukhin. 2017. Attention is all you need. In NIPS, pages 5998-6008.

Zeerak Waseem and Dirk Hovy. 2016. Hateful symbols or hateful people? predictive features for hate speech detection on twitter. In NAACL workshop, pages 88-93.

Yonghui Wu, Mike Schuster, Zhifeng Chen, Quoc V Le, Mohammad Norouzi, Wolfgang Macherey, Maxim Krikun, Yuan Cao, Qin Gao, Klaus Macherey, et al. 2016. Google's neural machine translation system: Bridging the gap between human and machine translation. arXiv preprint arXiv:1609.08144.

Ellery Wulczyn, Nithum Thain, and Lucas Dixon. 2017. Ex machina: Personal attacks seen at scale. In $W W W$, pages 1391-1399.

Marcos Zampieri, Shervin Malmasi, Preslav Nakov, Sara Rosenthal, Noura Farra, and Ritesh Kumar. 2019. Semeval-2019 task 6: Identifying and categorizing offensive language in social media (offenseval). In SemEval.

Yizhe Zhang, Siqi Sun, Michel Galley, Yen-Chun Chen, Chris Brockett, Xiang Gao, Jianfeng Gao, Jingjing Liu, and Bill Dolan. 2020. Dialogpt: Large-scale generative pre-training for conversational response generation. In $A C L$.

Ziqi Zhang and Lei Luo. 2019. Hate speech detection: A solved problem? the challenging case of long tail on twitter. Semantic Web, 10(5):925-945.

Ziqi Zhang, David Robinson, and Jonathan Tepper. 2018. Detecting hate speech on twitter using a convolution-gru based deep neural network. In $E S W C$, pages 745-760. 


\section{A Appendix}

\section{A.1 Parameter Estimation for pretraining HABERTOR with language model tasks}

Given the following input sentences $s_{i}=\left[w_{1}^{(i)}, w_{2}^{(i)}, \ldots, w_{u}^{(i)}\right]$ and $s_{j}=\left[w_{1}^{(j)}\right.$, $\left.w_{2}^{(j)}, \ldots, w_{v}^{(j)}\right]$, let the text sequence be $c_{l}=s_{i j}=$ $\left[w_{1}^{(i)}, w_{2}^{(i)}, \ldots, w_{u}^{(i)}, w_{1}^{(j)}, w_{2}^{(j)}, \ldots, w_{v}^{(j)}\right]=\left[w_{1}, \ldots, w_{n}\right]$ $(n=u+v)$ with label $y_{l}$ where we already paired the sentences to generate a next (i.e $y_{l}=1$ ) or not next (i.e. $y_{l}=0$ ) training instance. Let $\bar{c}_{l}$ be a corrupted sequence of $c_{l}$, where we masked some tokens in $c_{l}$. Denote $C$ a collection of such training text sequences $c_{l}$. The masked token prediction task aims to reconstruct each $c_{l} \in C$ given the corrupted sequence $\bar{c}_{l}$. In another word, the masked token prediction task maximizes the following log-likelihood:

$$
\begin{aligned}
\mathcal{L}_{1} & =\underset{\theta}{\operatorname{argmax}} \sum_{l=1}^{|\boldsymbol{C}|} \log p_{\theta}\left(c_{l} \mid \bar{c}_{l}\right) \\
& \approx \sum_{l=1}^{|\boldsymbol{C}|} \sum_{t=1}^{n} \mathbb{1}_{t} \log p_{\theta}\left(w_{t} \mid \bar{c}_{l}\right)
\end{aligned}
$$

where $\mathbb{1}_{t}$ is an indicator function and $\mathbb{1}_{t}=1$ when the token $t^{\text {th }}$ is a $[M A S K]$ token, otherwise $\mathbb{1}_{t}=$ 0 . $\theta$ refers to all the model's learning parameters, $w_{t}$ is the ground truth token at position $t^{t h}$. Denote $H_{\theta}\left(c_{l}\right)=\left[H_{\theta}\left(c_{l}\right)_{1}, H_{\theta}\left(c_{l}\right)_{2}, \ldots, H_{\theta}\left(c_{l}\right)_{n}\right]$ as the sequence of transformed output embedding vectors obtaining at the final layer of corresponding $n$ tokens in the sequence $c_{l} . H_{\theta}\left(c_{l}\right)_{t} \in \mathcal{R}^{d}$ with $d$ is the embedding size. By parameterizing a linear layer with a transformation $W_{1} \in \mathcal{R}^{V \times d}$ (with $V$ refers to the vocabulary size) as a decoder, we can rewrite $\mathcal{L}_{1}$ as follows:

$\mathcal{L}_{1}=\underset{\theta}{\operatorname{argmin}}-\sum_{i=1}^{|C|} \sum_{t=1}^{n} \mathbb{1}_{t} \log \frac{\exp \left(\left[W_{1} H_{\theta}\left(\bar{c}_{l}\right)_{t}\right]_{t}\right)}{\sum_{k=1}^{V} \exp \left(\left[W_{1} H_{\theta}\left(\bar{c}_{l}\right)_{t}\right]_{k}\right)}$

where $[\cdot]_{t}$ refers to the output value at position $t$.

For the next sentence prediction task, the objective is to minimize the following binary cross entropy loss function:

$$
\begin{aligned}
\mathcal{L}_{2}=\underset{\theta}{\operatorname{argmin}}-\sum_{i=1}^{|C|} y_{l} \log \left(\sigma\left(W_{2} H_{\theta}\left(c_{l}\right)_{1}\right)\right)+ \\
\left(1-y_{l}\right) \log \left(\sigma\left(W_{2} H_{\theta}\left(c_{l}\right)_{1}\right)\right)
\end{aligned}
$$

where $W_{2} \in \mathcal{R}^{d}$ and $H_{\theta}\left(c_{l}\right)_{1}$ refers to the embedding vector of the first token in the sequence $c_{l}$, or the [CLS] token. The intuition behind this is that the [CLS]'s embedding vector summarizes information of all other tokens via the attention Transformer network (Vaswani et al., 2017).

Then, pretraining with two language modeling tasks aims to minimize both loss functions $\mathcal{L}_{1}$ and $\mathcal{L}_{2}$ by: $\mathcal{L}_{L M}=\operatorname{argmin}_{\theta}\left(\mathcal{L}_{1}+\mathcal{L}_{2}\right)$

\section{A.2 Quaternion}

In mathematics, Quaternions ${ }^{5}$ are a hypercomplex number system. A Quaternion number $P$ in a Quaternion space $\mathbb{H}$ is formed by a real component $(r)$ and three imaginary components as follows:

$$
P=r+a \boldsymbol{i}+b \boldsymbol{j}+c \boldsymbol{k},
$$

where $i j k=i^{2}=j^{2}=k^{2}=-1$. The noncommutative multiplication rules of quaternion numbers are: $\boldsymbol{i j}=\boldsymbol{k}, \boldsymbol{j} \boldsymbol{k}=\boldsymbol{i}, \boldsymbol{k} \boldsymbol{i}=\boldsymbol{j}, \boldsymbol{j i}=-\boldsymbol{k}$, $\boldsymbol{k} \boldsymbol{j}=-\boldsymbol{i}, \boldsymbol{i} \boldsymbol{k}=-\boldsymbol{j}$. In Equa (4), $r, a, b, c$ are real numbers $\in \mathbb{R}$. Note that $r, a, b, c$ can also be extended to a real-valued vector $\in \mathbb{R}$ to obtain a Quaternion embedding, which we use to represent each word-piece embedding.

Algebra on Quaternions: We present the Hamilton product on Quaternions, which is the heart of the linear Quaternion-based transformation. The Hamilton product (denoted by the $\otimes$ symbol) of two Quaternions $P \in \mathbb{H}$ and $Q \in \mathbb{H}$ is defined as:

$$
\begin{aligned}
P \otimes Q= & \left(r_{P} r_{Q}-a_{P} a_{Q}-b_{P} b_{Q}-c_{P} c_{Q}\right)+ \\
& \left(r_{P} a_{Q}+a_{P} r_{Q}+b_{P} c_{Q}-c_{P} b_{Q}\right) \boldsymbol{i}+ \\
& \left(r_{P} b_{Q}-a_{P} c_{Q}+b_{P} r_{Q}+c_{P} a_{Q}\right) \boldsymbol{j}+ \\
& \left(r_{P} c_{Q}+a_{P} b_{Q}-b_{P} a_{Q}+c_{P} r_{Q}\right) \boldsymbol{k}+
\end{aligned}
$$

Activation function on Quaternions: Similar to (Tay et al., 2019; Parcollet et al., 2019), we use a split activation function because of its stability and simplicity. Split activation function $\beta$ on a Quaternion $P$ is defined as:

$$
\beta(P)=f(r)+f(a) \boldsymbol{i}+f(b) \boldsymbol{j}+f(c) \boldsymbol{k}
$$

, where $f$ is any standard activation function for Euclidean-based values.

Why does a linear Quaternion transformation reduce $75 \%$ of parameters compared to the linear Euclidean transformation? Figure 3 shows a comparison between a traditional linear Euclidean transformation and a linear Quaternion-based transformation.

In Euclidean space, the same input will be multiplied with different weights to produce different

\footnotetext{
${ }^{5}$ https://en.wikipedia.org/wiki/Quaternion
} 
Linear Euclidean transformation

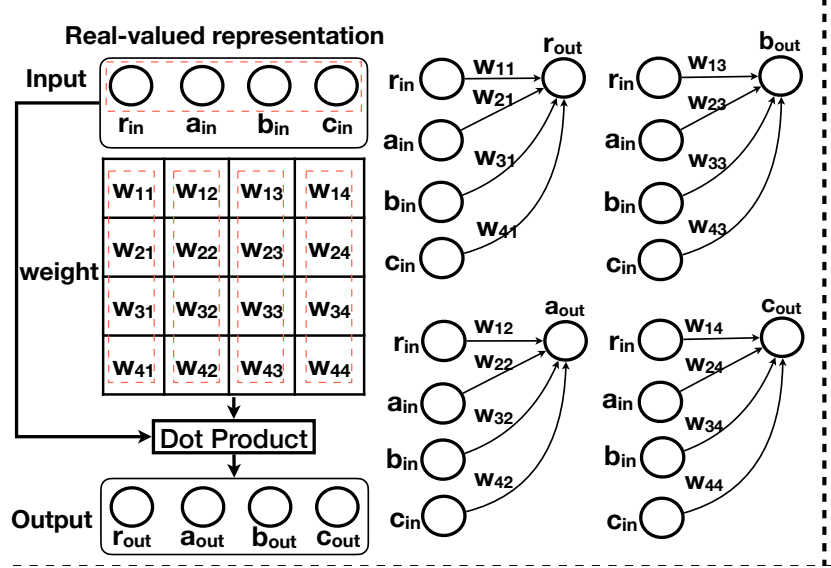

Linear Quaternion transformation Quaternion representation

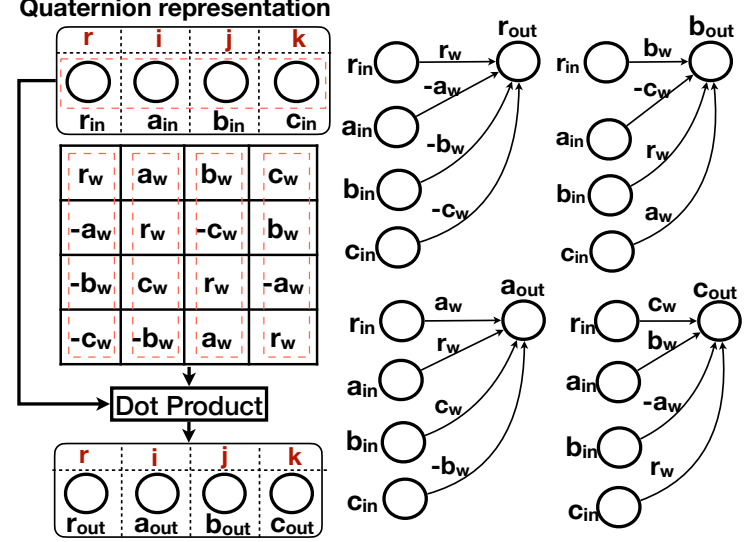

Figure 3: Comparison between linear Euclidean transformation (Left) and linear Quaternion transformation (Right). The Hamilton product in Quaternion space is replaced with an equivalent dot product in real space for an easy reference. Computing each output dimension in real-valued transformation (left) always need 4 new parameters, resulting in 16 degrees of freedom. In contrast, only 4 parameters are used and shared in producing all 4 output dimensions in Quaternion transformation, leading to a better inter-dependency encoding and a $75 \%$ of parameter saving.

output dimensions. Particularly, given a real-valued 4-dimensional vector $\left[r_{i n}, a_{i n}, b_{i n}, c_{i n}\right]$, we need to parameterize a weight matrix of 16 parameters (i.e. 16 degrees of freedom) to transform the 4dimensional input vector into a 4-dimensional output vector $\left[r_{\text {out }}, a_{\text {out }}, b_{\text {out }}, c_{\text {out }}\right]$. However, with Quaternion transformation, the input vector now is represented with 4 components, where $r_{i n}$ is the value of the real component, $a_{i n}, b_{i n}, c_{i n}$ are the corresponding value of the three imaginary parts $i$, $j, k$, respectively. Because of the weight sharing nature of Hamilton product, different output dimensions take different combinations with the same input with exactly same 4 weighting parameters $\left\{r_{w}, a_{w}, b_{w}, c_{w}\right\}$. Thus, the Quaternions transformation reduces $75 \%$ of the number of parameters compared to the real-valued representations in $\mathrm{Eu}-$ clidean space.

Quaternion-Euclidean conversion: Another excellent property of using Quaternion representations and Quaternion transformations is that converting from Quaternion to Euclidean and vice versa are convenient. To convert a real-valued based vector $v \in \mathcal{R}^{d}$ into a Quaternion-based vector, we consider the first $d / 4$ dimensions of $v$ as the value of the real component, and the corresponding next three $d / 4$ dimensions are for the three imaginary parts, respectively. Similarly, to convert a Quaternion vector $v \in \mathcal{H}^{d}$ into a real-valued vector, we simply concatenate all four components of the Quaternion vector, and treat the concatenated vector as a real-valued vector in Euclidean space.

\section{A.3 Analysis on the BERT's Parameters}

Figure 4 presents a general view of the BERT architecture. Each BERT layer contains three parts: (i) attention, (ii) filtering, and (iii) output.

The attention part parameterizes three weight transformation matrices $\mathrm{H} \times \mathrm{H}$ to form key, query, and value from the input, and another weight matrix $\mathrm{H} \times \mathrm{H}$ to transform the output attention results. The total parameters of this part are $4 H^{2}$. The filtering part parameterizes a weight matrix $\mathrm{H} \times 4 \mathrm{H}$ to transform the output of the attention part, leading to a total of $4 H^{2}$ parameters. The output part parameterizes a weight matrix $4 \mathrm{H} \times \mathrm{H}$ to transform the output of the filtering part from $4 \mathrm{H}$ back to $\mathrm{H}$, resulting in $4 H^{2}$ parameters.

Thus, a BERT layer has $12 H^{2}$ parameters, and a BERT-base setting with 12 layers has $144 H^{2}$ parameters. By taking into account the number of parameters for encoding $\mathrm{V}$ vocabs, the total parameters of BERT is $\mathrm{VH}+144 H^{2}$.

\section{A.4 50-50 Rule}

To ensure the 50-50 rule, we perform the following method: Let $\mathrm{M}$ be the number of input text sequences that we can split into multiple sentences, and $\mathrm{N}$ be the number of input sequences that can be tokenized into only one sentence. We want the number of sentences to be generated as next sentence pairs (sampling with probability $p_{1}$ ) to be roughly equal to the number of sentences to be formed as not next sentence pairs (sampling with probability $p_{2}$ ). In another word, 


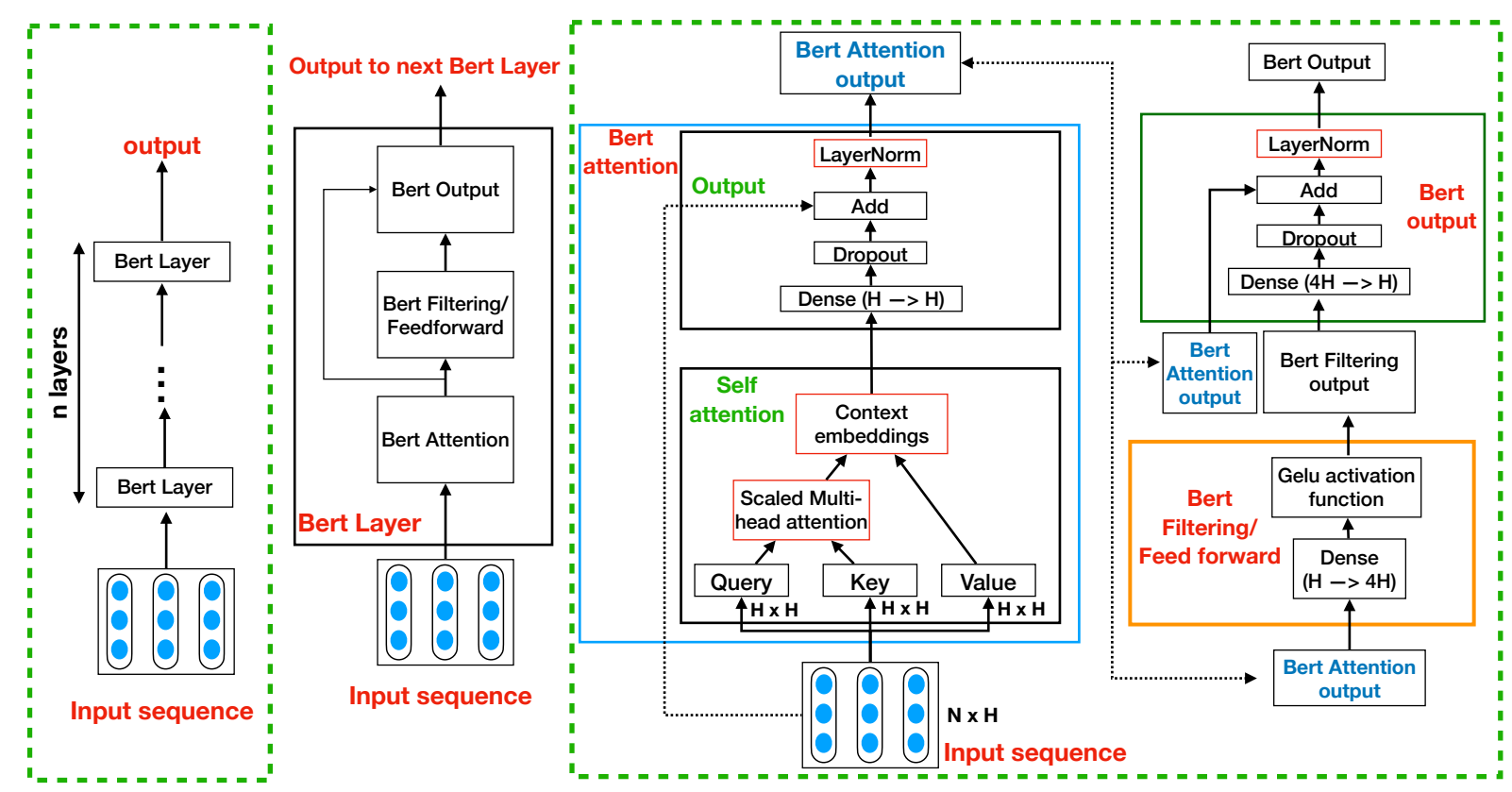

Figure 4: General view of the BERT architecture. Uncovering the architecture from left to right.

$M \times p_{1}=(M+N) \times p_{2}$ or $\frac{p_{1}}{p_{2}}=\frac{(M+N)}{M}$. Since $p_{1}+p_{2}=1$, replacing $p_{2} \stackrel{p_{2}}{=} 1-p_{1}$, we have: $M \times p_{1}=(M+N)\left(1-p_{1}\right) \longrightarrow p_{1}=\frac{(M+N)}{(2 M+N)}$ With $p_{1}$ established, we set $p_{1}$ as the probability for a sentence to be paired with another consecutive sentence in a same input sequence to generate a next sentence example.

\section{A.5 Baselines and Hyper-parameter Settings}

15 Baselines are described as follows:

- BOW: Similar to Dinakar et al. (2011); Van Hee et al. (2015), we extract bag of words features from input sequences, then a traditional machine learning classifier is built on top of the extracted features.

- NGRAM: It is similar to BOW model, except using n-gram features of the input sequence.

- CNN (Kim, 2014): It is a state-of-the-art word based CNN neural network model.

- VDCNN (Conneau et al., 2017): It is a character based CNN model with a deeper architecture and optional shortcut between layers.

- FastText (Joulin et al., 2016): An extension of the Word2 Vec model, where it represents each word as an n-gram of characters to provide embeddings for rare words.

- LSTM (Cho et al., 2014): We use: (i) the last LSTM output vector, and (ii) a pooling layer (max and mean) to aggregate LSTM output vectors and report only the best results.
- att-LSTM: A LSTM model with an attention layer to aggregate LSTM hidden state vectors.

- RCNN (Lai et al., 2015): A combination between a bi-directional recurrent structure to capture contextual information and a max pooling layer to extract key features.

- att-BiLSTM (Lin et al., 2017): It is a selfattentive Bidirectional LSTM model.

- Fermi (Indurthi et al., 2019): The best hatespeech detection method, as reported in (Basile et al., 2019). It built a SVM classifier on top of the pretrained embeddings from Universal Sentence Encoder (USE) (Cer et al., 2018) model.

- Q-Transformer (Tay et al., 2019): It is a Quaternion Transformer. It replaces all Euclidean embeddings and linear transformations by Quaternion emddings and Quaternion linear transformation. We use the full version of Q-Transformer due to its high effectiveness.

- Tiny-BERT (Jiao et al., 2019): It is a compressed model of BERT-base by performing knowledge distillation on BERT-base during its pretraining phase with smaller number of layers and embedding sizes. We adopt the Tiny-BERT 4 layers with $14.5 \mathrm{M}$ of parameters.

- DistilBERT-base (Sanh et al., 2019): another knowledge distillation of the BERT-base model during the BERT's pre-training phase.

- ALBERT-base (Lan et al., 2020): a light- 
weight version of BERT-base model with parameters sharing strategies and an intersentence coherence pretraining task.

- BERT-base (Devlin et al., 2019): Similar to Nikolov and Radivchev (2019), we use pretrained BERT with 12 layers and uncased (our experiments show uncased works better than cased vocab) to perform fine-tuning for the hatespeech detection.

For baselines that require word embeddings, to maximize their performances, we initialize word embeddings with both GloVe pre-trained word embeddings (Pennington et al., 2014) and random initialization and report their best results. We implement BOW and NGRAM with Naive Bayes, Random Forest, Logistic Regression, and Xgboost classifiers, and then report the best results.

By default, our vocab size is set to $40 \mathrm{k}$. The number of pretraining epochs is set to 60 , and the batch size is set to 768 . The learning rate is set to $5 \mathrm{e}-5$ for the masked token prediction and next sentence prediction tasks, which are the two pretraining tasks, and 2e-5 for the hatespeech prediction task, which is the fine-tuning task. The default design of HABERTOR is given at Figure 1b, with one separated classification net with an ensemble of 2 heads for each input source. The masking factor $\tau$ is set to 10. The noise magnitude's bound constraint $[a, b]=[1,2]$ in Yahoo dataset, and $[a, b]=[1,5]$ in Twitter and Wiki datasets. $\lambda_{a d v}=1.0$, and $\lambda_{\epsilon}=1$ in all three datasets. We use $\min$ pooling function to put a more stringent requirement on classifying hatespeech comments, as the number of hatespeech-labeled comments is the minority. All the pre-trained language models are fined-tuned with the Yahoo train set. For all other baselines, we vary the hidden size from $\{96,192,384\}$ and report their best results. We build VDCNN with 4 convolutional blocks, which have $64,128,256$ and 512 filters with a kernel size of 3, and 1 convolution layer. Each convolutional block includes two convolution layers. For FastText, we find that 1,2,3-grams and 1,2,3,4,5-character grams give the best performance. All models are optimized using Adam optimizer (Kingma and Ba, 2014).

\section{A.6 Ablation Study}

Effectiveness of regularized adversarial training and masking factor $\tau$ (AS1): Recall that by default, HABERTOR has 2 classification nets, each of the two nets has an ensemble of 2 classification heads, masking factor $\tau=10$, and is trained with regularized adversarial training. HABERTOR $a d v$ indicates HABERTOR without regularized adversarial training, and HABERTOR $-a d v+\tau=1$ indicates HABERTOR without regularized adversarial training and masking factor $\tau$ of 1 instead of 10. Comparing HABERTOR with HABERTOR - $a d v$, we see a drop of AP by $1.16 \%$, F1-score by $1.16 \%$, and the average error rate increases by $0.78 \%$ (i.e. average of FPR@5\%FNR and FNR@5\%FPR). This shows the effectiveness of additional regularized adversarial training to make HABERTOR more robust. Furthermore, comparing HABERTOR - $a d v$ (with default $\tau=10$ ) with HABERTOR $-a d v+\tau=1$, we observe a drop of AP by $0.92 \%$, F1-score by $0.24 \%$, and an increment of average error rate by $1.01 \%$. This shows the need of both regularized adversarial training with our proposed fine-grained and adaptive noise magnitude, and a large masking factor in HABERTOR.

Is pretraining with a larger domain-specific dataset helpful? (AS2): We answer the question by answering a reverse question: does pretraining with smaller data reduce performance? We pre-train HABERTOR with 250k Yahoo comments data (4 times smaller), and 500k Yahoo comments data ( 2 times smaller). Then, we compare the results of HABERTOR $-a d v+\tau=1$ with HABERTOR $-a d v+\tau=1$ under 250k data, and HABERTOR $-a d v+\tau=1$ under 500k data. Table 7 shows the results. We observe that pretraining with a larger data size increases the hatespeech prediction performance. We see a smaller drop when pretraining with $1 \mathrm{M}$ data vs $500 \mathrm{k}$ data (AP drops $0.6 \%$ ), and a bigger drop when pretraining with 500k data vs $250 \mathrm{k}$ data (AP drops $4.4 \%$ ). We reason that when the pretraining data size is too small, important linguistic patterns that may exist in the test set are not fully observed in the training set. In short, pretraining with larger hatespeech data can improve the hatespeech prediction performance. Note that BERT-base is pre-trained on 3,300M words, which are 106 times larger than HABERTOR (only 31M words). Hence, the performance of HABERTOR can be boosted further when pre-training a hatespeech language model with a larger number of hateful representatives.

Usefulness of separated source prediction and ensemble heads (AS3): We compare HABERTOR from Default settings to using single source 
Table 7: Ablation study of HABERTOR on Yahoo dataset (i.e. both Yahoo News + Finance, to save space). Default results are in bold. Better results compared to the default one are underlined.

\begin{tabular}{|c|c|c|c|c|c|c|}
\hline \multirow[b]{2}{*}{ Goal } & \multirow{2}{*}{ Model } & \multicolumn{5}{|c|}{ Yahoo } \\
\hline & & AUC & $\mathrm{AP}$ & $\begin{array}{c}\text { FPR@ } \\
5 \% \text { FNR }\end{array}$ & $\begin{array}{l}\text { FNR@ } \\
5 \% \text { FPR }\end{array}$ & $\mathrm{F} 1$ \\
\hline & Default & 94.77 & 72.35 & 26.11 & 25.08 & 66.18 \\
\hline \multirow{2}{*}{ AS1 } & $-\operatorname{adv}$ & 94.60 & 71.19 & 26.97 & 25.78 & 65.02 \\
\hline & $-\operatorname{adv}+\tau=1$ & 94.32 & 70.27 & 28.08 & 26.69 & 64.78 \\
\hline \multirow{2}{*}{ AS2 } & $-\operatorname{adv}+\tau=1$ under $250 \mathrm{k}$ data & 92.61 & 64.71 & 36.43 & 32.51 & 60.13 \\
\hline & $-\operatorname{adv}+\tau=1$ under $500 \mathrm{k}$ data & 94.04 & 69.11 & 29.82 & 27.99 & 63.34 \\
\hline \multirow{4}{*}{ AS3 } & + single source + single head & 94.70 & 71.82 & 26.82 & 25.55 & 65.16 \\
\hline & + single head & 94.70 & 72.15 & 26.66 & 25.20 & 65.59 \\
\hline & + ensemble 4 & 94.78 & 72.18 & 26.29 & 24.97 & 65.78 \\
\hline & + ensemble 8 & $\overline{94.71}$ & 72.06 & 26.13 & $\overline{25.08}$ & 65.56 \\
\hline AS4 & $-\operatorname{adv}+\tau=1$ - pretraining & 92.48 & 65.26 & 36.47 & 32.10 & 60.66 \\
\hline \multirow{6}{*}{ AS5 } & +3 layers & 94.54 & 71.25 & 27.15 & 25.90 & 64.98 \\
\hline & +4 layers & 94.67 & 71.53 & 26.25 & 25.50 & 65.38 \\
\hline & + 192 hidden size & 94.57 & 71.00 & 26.56 & 25.93 & 65.05 \\
\hline & +3 att heads & 94.69 & 72.00 & 26.72 & 25.43 & 65.75 \\
\hline & +4 att heads & 94.69 & 72.06 & 26.61 & 25.22 & 65.80 \\
\hline & +12 att heads & 94.70 & 72.01 & 26.28 & 25.14 & 65.64 \\
\hline
\end{tabular}

+ single source (i.e. one classification head for all data sources, see Figure 1a), single head (i.e. multisource and each source has a single classification head, see Figure 1b), and using more ensemble heads (i.e. multi-source + more ensemble classification heads, see Figure 1b). Table 7 shows that the overall performance order is multi-source + ensemble of 2 heads $>$ multi-source + single head $>$ single source + single head, indicating the usefulness of our multi-source and ensemble of classification heads architecture in the fine-tuning phase. However, when the number of ensemble heads $\geq$ 4 , we do not observe better performance.

Is pretraining two language modeling tasks helpful for the hatespeech detection task? (AS4) We compare HABERTOR- $a d v+\tau=1$ with HABERTOR- $a d v+\tau=1$ - pretraining, where we ignore the pretraining step and consider HABERTOR as an attentive network for pure supervised learning with random parameter initialization. In Table 7, the performance of HABERTOR without the language model pretraining is highly downgraded: AUC drops $\sim-2 \%$, AP drops $\sim-5 \%$, FPR and FNR errors are $\sim+9 \%$ and $\sim+5 \%$ higher, respectively, and F1 drops $-4 \%$. These results show a significant impact of the pretraining tasks for hatespeech detection.

Is HABERTOR sensitive when varying its number of layers, attention heads, and embedding size? (AS5) In Table 7, we observe that HABER$\mathrm{TOR}+3$ layers and HABERTOR+4 layers work worse than HABERTOR (6 layers), indicating that a deeper model does help to improve hatespeech detection. However, when we increase the number of attention heads from 6 to 12 , or decrease the number of attention heads from 6 to 4 , we observe that the performance becomes worse. We reason that when we set the number of attention heads to 12 , since there is no mechanism to constrain different attention heads to attend on different information, they may end up focusing on the similar things, as shown in (Clark et al., 2019). But when reducing the number of attention heads to 4 , the model is not complex enough to attend on more relevant information, leading to worse performance. Similarly, when we reduce the embedding size from 384 in HABERTOR to 192, the performance is worse. Note that we could not perform experiments with larger embedding sizes and/or more number of layers due to high running time and memory consumption. However, we can see in Table 7 performance of smaller HABERTOR with 3 layers, 4 layers, or 192 hidden size still obtain slightly better than BERT-base results reported in Table 3. This again indicates the need for pretraining language models on hatespeech-related corpus for the hatespeech detection task.

\section{Effectiveness of fine-grained pretraining}




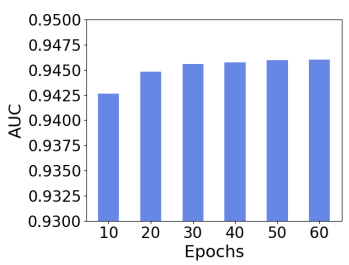

(a) AUC.

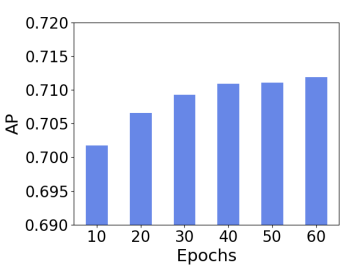

(b) AP.

Figure 5: AUC and AP of HABERTOR without regularized adversarial training on Yahoo dataset when varying the number of epochs for the pretraining task.

(AS6)? Since the pretraining phase is unsupervised, a question is how much fine-grained pretraining should we perform to get a good hatespeech prediction performance? Or how many pretraining epochs are good enough? To answer the question, we vary the number of the pretraining epochs from $\{10,20,30, \ldots, 60\}$ before performing the fine-tuning phase with hatespeech classification task. We report the changes in AUC and AP of fine-tuned HABERTOR on the Yahoo dataset without performing regularized adversarial training in Figure 5. We observe that a more fine-grained pretraining helps to increase the hatespeech prediction results, which is similar to a recent finding at Liu et al. (2019), especially from 10 epochs to 40 epochs. However, after 40 epochs, the improvement is smaller. 